\title{
Review of: "Multicenter study evaluating novel multi- specimen pooling assay for the detection of SARS- CoV-2: High sensitivity and high throughput testing"
}

Robert Paulino-Ramirez ${ }^{1}$

1 Universidad Iberoamericana

Potential competing interests: The author(s) declared that no potential competing interests exist.

The purpose of this study was to evaluate screening assay for SARS-CoV-2 to facilitate an increased number of analyzed samples processed while increasing testing capacity without affecting the accuracy and sensitivity. Researchers then analyzed a pooling testing strategy of $6 p$ (one positive to five negative specimens in one tube) in approximately $2.5 \mathrm{hrs}$ that showed a higher throughput than standardized rRTPCR assays with an limit of detection (LoD) of 1000 copies $/ \mathrm{ml}$ and an overall percentage agreement between methods for the 330 samples examined was $100 \%$ in positive specimens for low, medium, and high Ct value.

LoD assessment based on a on the LabTurbo AIO 48 platform, an automated platform that allows nucleic acid extraction and sample analysis targeting N1 and E gene on the same instrument and compared to the current WHO standardized method of detection based on qRT-PCR reaction. Assessing first, the limit of detection using serial dilutions of RNA controls of total volumes of 300, 900, 1500, 1800 and $2100 \mathrm{ul}$ respectively, to conclude that six-in-one was the clinical pooling strategy performing with a higher sensitivity at 1000 copies. This finding is similar to a study performed in the Dominican Republic by Tejada Ramirez et al, 2021 where $4 p$ where considered the appropriate sample strategy to avoid wasting lab resources (1).

The use of an automated system that integrates RNA extraction and qRT-PCR analysis with sample-toresult capability can be very effective and are ideally suited for laboratories that may not be suitable with high-complexity molecular testing methods.

Sample pooling strategies can be very effective when resources are limited yet it comes with its own limitations. The researchers proposed an expansion of the volume limit, but when samples are diluted, there could be less viral genetic material and a higher variability at a higher number of pooled samples which increases the likelihood of false negatives. Testing limitations such as an incorrectly taken sample, contaminated sample, or even time of collection should also be taken into consideration.

Pool testing is a viable option as a surveillance technique and for sample analysis in a manner that represents a rational use of resources, but it is a strategy that is effective at a low incidence rates in the context of SARS-CoV-2. 


\section{References}

1. Ramírez MT, Rosario CD, Contreras E, Cabrera J, Degaudenzi AV, Ramírez RP. Evaluation of sample pooling for the detection of SARS-CoV-2 in a resource-limited setting, Dominican Republic. Enferm Infecc Microbiol Clin (Engl Ed). 2021 Jul 26. doi: 10.1016/j.eimc.2021.07.004. Epub ahead of print. PMID: 34334859; PMCID: PMC8310719. 